\title{
VALPROIC ACID TOXICITY INDUCED BY LACOSAMIDE: A RARE CASE REPORT
}

\author{
KARTHIKA ASHOK KUMAR, CHANSHI CHANDRAN, REMYA REGHU*
}

Department of Pharmacy Practice, Amrita School of Pharmacy, Amrita University, Kochi - 682 041, Kerala, India.

Email: remyareghu @aims.amrita.edu

Received: 31 March 2017, Revised and Accepted: 20 May 2017

ABSTRACT

Valproic acid is commonly used in the treatment of both focal and generalized epilepsies and is often tolerated well. Valproic acid is usually tolerated well, but serious complications, including hepatotoxicity and hyperammonemic encephalopathy, may occur. Here, we report a case of valproic acidinduced hyperammonemic encephalopathy on concomitant use with lacosamide.

Keywords: Valproic acid, Hyperammonemic encephalopathy, Lacosamide.

(c) 2017 The Authors. Published by Innovare Academic Sciences Pvt Ltd. This is an open access article under the CC BY license (http://creativecommons. org/licenses/by/4. 0/) DOI: http://dx.doi.org/10.22159/ajpcr.2017.v10i9.18820

\section{INTRODUCTION}

Valproic acid is a branched-chain carboxylic acid and is mainly used in the treatment of epilepsy and bipolar disorder. It is a broad-spectrum antiepileptic agent used in the treatment of absence, myoclonic, partial, and tonic-clonic seizures $[1,2]$ It is also used to prevent migraine headaches. It acts by increasing the availability of gamma-aminobutyric acid (GABA), an inhibitory neurotransmitter, to brain neurons or may enhance the action of GABA or mimic its action at post-synaptic receptor sites [3]. The most common side effects are transient gastrointestinal side effects including anorexia, nausea, and vomiting in approximately $16 \%$ of patients. It is usually tolerated well, but serious complications, including hepatotoxicity and hyperammonemic encephalopathy, may occur. Hyperammonemic encephalopathy is seen rarely, but potentially fatal adverse drug reaction to valproic acid [4]. It can occur when using valproic acid as monotherapy, but it more commonly occurs when using antiepileptics as combination therapy. Prior reports are there implicating phenytoin and phenobarbital as contributing to valproic acid hyperammonemic encephalopathy, but newer antiepileptic agents are less frequently implicated [5]. Lacosamide is used as an adjunctive treatment for epilepsy [6]. We present a rare case report of valproate toxicity, that is, hyperammonemic encephalopathy induced by concomitant lacosamide use for the treatment of status epilepticus.

\section{CASE REPORT}

A 23-year-old male patient is a known case of status epilepticus, with a history of seizures since neonatal period with normal development but not good in academics. He was on carbamazepine $200 \mathrm{mg}$ twice daily, valproate $400 \mathrm{mg}$ twice daily, and escitalopram $0.5 \mathrm{mg}$ twice daily. He has a baseline seizure frequency of once in every 2 weeks with semiology of up rolling of eyes with tonic posturing of upper and lower limbs which last less than a minute with post-ictal drowsiness of about half and hour. He was brought to the tertiary care teaching hospital with 8 episodes of seizure with similar semiology, no regain of consciousness between the last 3-4 seizures. On examination, patient's vitals were stable, pupils bilaterally equally reacting to light, moving all four limbs, normal reflexes, and flexor plantars. The patient was started on continuous electroencephalogram monitoring and it revealed epileptiform discharges. The patient was given lacosamide loading dose of $200 \mathrm{mg}$ IV over 15 minutes and continued on maintenance dose of lacosamide 200 mg 1-0-1, then on next day, his serum ammonia level was found to be $120.8 \mathrm{umol} / \mathrm{L}$ (normal level: 10-47 umol/L) which was managed with l-carnitine. His symptoms resolved later and his serum ammonia levels came down. Valproate and lacosamide were discontinued and patient recovered fully.

\begin{tabular}{llll}
\hline Test & 16/9/16 4.23 PM & 16/9/16 8.45 PM 17/9/16 4.50 PM \\
\hline Serum & 120.8 umol/L & 38.7 umol/L & 37.6 umol/L \\
ammonia & & & \\
reference & & \\
range & & \\
$(10-47$ & & \\
umol/L) & & \\
\hline
\end{tabular}

\section{DISCUSSION}

Several case reports on valproate-induced hyperammonemia have been reported previously but very few reports on lacosamideinduced valproic acid toxicity were reported in the previous years. The patient was having seizure since neonatal period and he was on valproic acid since then. He never developed hyperammonemia in the past years. Addition of lacosamide seems to have promoted this effect because this was the recent medication given to the patient. Valproic acid is known to cause carnitine deficiency, which can ultimately lead to hyperammonemia as carnitine helps in the metabolism and elimination of valproic acid through beta-oxidation of fatty acids in the liver [7]. L-carnitine promotes the transport of long-chain fatty acids from the cytosol compartment of the muscle fiber to the mitochondria, they undergo $\beta$-oxidation and produce acetyl-CoA [8].

Discontinuation of the drug is the primary treatment of valproic acid toxicity. Full recovery is anticipated in most patients [9]. In symptomatic patients whose mental status is altered and is not due to seizure activity dosage adjustment or discontinuation of valproate may be necessary [10]. Mechanism by which lacosamide contributes to valproic acid toxicity is unclear, but this possibility should be considered in any patient on these antiepileptics who presents with altered mental status.

\section{CONCLUSION}

Valproic acid toxicity can be induced in the presence of lacosamide although it is not commonly seen. Physicians need to be aware of such cases; early diagnosis allows prompt withdrawal of the offending drug.

\section{ACKNOWLEDGMENT}

The authors are grateful to the patient and his family for giving consent to present this case report. 


\section{REFERENCES}

1. Crudup JB, Hartley BI, Keel BR, Mukta P. Recognizing and treating valproic acid toxicity: A case report. J Med Cases 2011;5:185-7.

2. Nampoothiri V, Lakshmi R. Valproate induced transaminitis. Int J Pharm Pharm Sci 2015;7(12):304-5.

3. Available from: http://www.uptodate.com/valproic acid drug information. [Last accessed on Mar 12].

4. Lheureux PE, Hantson P. Carnitine in the treatment of valproic acidinduced toxicity. Clin Toxicol (Phila) 2009;47(2):101-11.

5. Hamer H, Knake S, Schomburg U, Rosenow F. Valproate-induced hyperammonemic encephalopathy in the presence of topiramate. Neurology 2000;54:230-2.

6. Doty P, Rudd GD, Stoehr T, Thomas D. Lacosamide. Neurotherapeutics
2007;4(1):145-8

7. Raskind J, El-Chaar G. The role of carnitine supplementation during valproic acid therapy. Ann Pharmacother 2000;34(5):630-8.

8. Shaalan SH, El-Wakkad AS, Saleh HA, Deab A. Protective effect of L-carnitine and baker yeast saccharomyces cerevisiae against hepatic toxicity induced by valproate as antiepileptic drug in rats. Int J Pharm Pharm Sci 2015;7(5):89-95.

9. Gerstner T, Buesing D, Longin E, Bendl C, Wenzel D, Scheid B, et al. Valproic acid induced encephalopathy-19 new cases in Germany from 1994 to 2003-a side effect associated to VPA-therapy not only in young children. Seizure 2006;15(6):443-8.

10. Tarafdar S, Slee M, Ameer F, Doogue M. A case of valproate induced hyperammonemic encephalopathy. Case Rep Med 2011;2011:969505. 